\title{
Performance Comparison of different hybrid amplifiers for different numbers of channels
}

\author{
Sameksha Bhaskar ${ }^{1}$, M.L.Sharma ${ }^{2}$ \\ Department of Electronics and Communication, \\ BGIET, Sangrur, India
}

\author{
Ramandeep Kaur ${ }^{2}$ \\ Department of Electronics and Communication \\ THAPAR, Patiala, India
}

\begin{abstract}
We have investigated the performance comparison of different hybrid optical amplifiers (RAMAN-EDFA,RAMANSOA,SOA-EDFA,EDFA-RAMAN-EDFA).The proposed configuration consists of 16,32 and 64 Gbps channels at speed of 10 Gbps. We have realized the different hybrid amplifiers and their parameters like quality factor, ber, eye opening and jitter at different number of channels. The different combinations can provide a better result and better feasibility for long distance transmission. It is observed that SOA-EDFA showed good performance as it can travel max distance of $220,240,260 \mathrm{~km}$ at 16, 32 and 64 channels respectively. Also, RAMAN-EDFA showed a good performance as it has a high QUALITY FACTOR (24.27) and BER $\left(1 \times 10^{-40}\right)$ at 16 channels.
\end{abstract}

Keywords-RAMAN-EDFA; RAMAN-SOA; SOA-EDFA; EDFARAMAN-EDFA; QUALITY FACTOR; BER; EYE OPENING; JITTER.

\section{INTRODUCTION}

Technology developments have described that optical is fantastic for the transmission. In fiber optic communications, WDM is a technology which multiplexes a number of optical carrier signals onto a single optical fiber by using different wavelengths at speed in Gbps [1-3].WDM of optical signals is a promising way to increase transmission capacity of a fiber. The development of WDM has allowed us to exploit large amount of bandwidth available in optical fiber as low capacity channels [4]

An optical network is optical but switching could be optical, electrical or hybrid as well [5] whereas optical amplifiers are used which directly amplify the transmitter optical signal without conversion to electric forms and also can be used for long distance transmission which can be pre or post amplifier. An optical amplifier is a laser without a feedback [6]. It uses the principle of stimulated emission same as used in laser.

Dense wavelength division multiplexing (DWDM) uses the same transmission window but with denser channel spacing. Channel plans vary, but a typical system would use 40 channels at $100 \mathrm{GHZ}$ spacing or 80 channels with $50 \mathrm{GHZ}$ spacing. The cascading a semiconductor optical amplifier (SOA) and a fiber Raman amplifier (FRA or RA) is called hybrid amplifier. There are two kinds of hybrid amplifiers (HA): seamless and wideband hybrid amplifiers (SWB-HA) and narrowband amplifier (NA). Hybrid amplifier increases the transmitter power by placing it just after the transmitter and before the receiver without any splitters, boosters in between as it increases the noises and distortion.

A Carena et al. [7] investigated on the optimal configuration of hybrid Raman/Edfa yielding a closed form analysis. In order to compare different system configurations, impact of fiber non linearities has been introduced. The maximum reachable distance has been evaluated with a target signal to noise ratio. Also he demonstrated that Raman amplification, combined with EDFAs, allow the increase of the maximum reachable distance and/or the span length. Raman amplification can also be used to substantially reduce the impact of fiber nonlinearity.

Chieng Hung Yeh et al. [8] demonstrated a new hybrid three stage L-band fiber amplifier module composed of a semiconductor optical amplifier and two Edfa over gain bandwidth of 1540 to $1600 \mathrm{~nm}$. This proposed amplifier also provides a broadband amplified spontaneous emission light source. Also he experimentally demonstrated a new hybrid Lband fiber amplifier utilizing a semiconductor optical amplifier and two erbium-doped fiber amplifiers over the gain bandwidth of 1540 to $1600 \mathrm{~nm}$. This proposed amplifier also provides broadband ASE light source. Therefore, this amplifier is useful in application to WDM networks.

$\mathrm{Ju}$ Han Lee et al. [9]demonstrated on the performance of three different schemes of single pump dispersion compensating fiber based Raman/Edfa hybrid amplifier together with a DCF based Raman only amplifier in terms of static properties, dynamic and system impact. With respect to overall gain and system impact, based on BER hybrid amplifier was found to have the best performance among other types. On the contrary, a much higher transient tolerance was observed in the Raman-only amplifier than the hybrid amplifiers since the EDF section in the three types of hybrid amplifiers is highly sensitive to transient environments.

Seung Kwan Kim et al. [10] proposed the design of hybrid amplifier composed of a distributed Raman amplifier and Edfa. They characterize the distributed Raman amplifier by numerical simulation based on measured Raman gain coefficient of single mode fiber. They estimated the performance of the hybrid amplifier for long haul optical transmission. In single channel amplification, the crosstalk caused by double Rayleigh scattering was independent of signal input power and simply given as a function of the Raman gain. Compared with erbium-doped fiber amplifiers, the 
optical signal-to-noise ratio was calculated to be higher by more than $3 \mathrm{~dB}$ in the optical link using the designed hybrid amplifier

T. Sakamoto et al. [11] designed the gain characteristics of the hybrid fiber amplifier that consists of cascaded thulium doped fiber amplifiers and Edfa are reported. The results showed that the hybrid amplifier have a gain of over $20 \mathrm{~dB}$ with bandwidth of $20 \mathrm{Db}$ and wavelength range of about 1460 and $1560 \mathrm{~nm}$. The low noise figure (NF) below $7 \mathrm{~dB}$ was obtained in 1460-1540 nm when placing a TDFA in the first stage followed by an EDFA and in 1480-1560 nm when placing amplifiers in a reversed order. The gain-equalization technique was applied, and the hybrid amplifier that had an average gain of $20 \mathrm{~dB}$, a gain excursion of less than $2 \mathrm{~dB}$, an output power of $14.5 \mathrm{dbm}$, and an NF of less than $7 \mathrm{~dB}$ in the 77-nm gain band was achieved.

We pursue and extend the same work which includes comparison of only 16 channel whereas we have compared the performances of different hybrid amplifiers on various number of channels of 16, 32 and 64 .

This paper is organized into four sections. We focus on the comparison and performance of hybrid amplifiers. In section 2, simulation setup for comparison of different hybrid amplifiers. The performance of amplifiers is analyzed here. Section 3 gives the discussions of results observed after simulation. Section 4 gives the conclusion of Quality Factor, Ber, Eye Opening, Jitter, and Power at different channels.

\section{Simulation SetUP}

To investigate the performance of the hybrid amplifiers, at sixteen, thirty two or sixty four user transmitters are used at a speed of $10 \mathrm{Gbps}$ speed as shown in fig 1. The signals from data source and laser are fed to the external Mach-Zehnder modulator, where the input signals from data source are modulated through a carrier optical output signal is transmitted. These signals are transmitted over spliter to which the optical power meter and optical spectrum analyser is attached. The optical splitter splits the optical signals into two or more outputs. Further a compound component is placed which consists of Raman-Edfa, Raman-Soa, Soa-Edfa, and EdfaRaman-Edfa. We can also choose any other hybrid amplifiers according to the requirements. Again then optical splitter is attached to which optical power meter and optical spectrum analyser are attached. Later, the receiver is attached which is used to detect all signals and converted into electrical signal. Different types of optical amplifiers are applied at different channels. The optical signal is transmitted and measured over different distance using different number of channels. The different parameters like quality factor, Ber, eye opening, jitter are calculated at different channels and best of it is calculated at various channels. Optical signals are amplified using EDFA amplifier. The signal power is measured by power meter and optical probe. The modulated signal is converted into original signal with the help of PIN photodiode and filters. A compound receiver is used to detect all the signals and converts these into electrical signals. Also a power meter can be attached to achieve the power at the receiver end which is needed for the project.

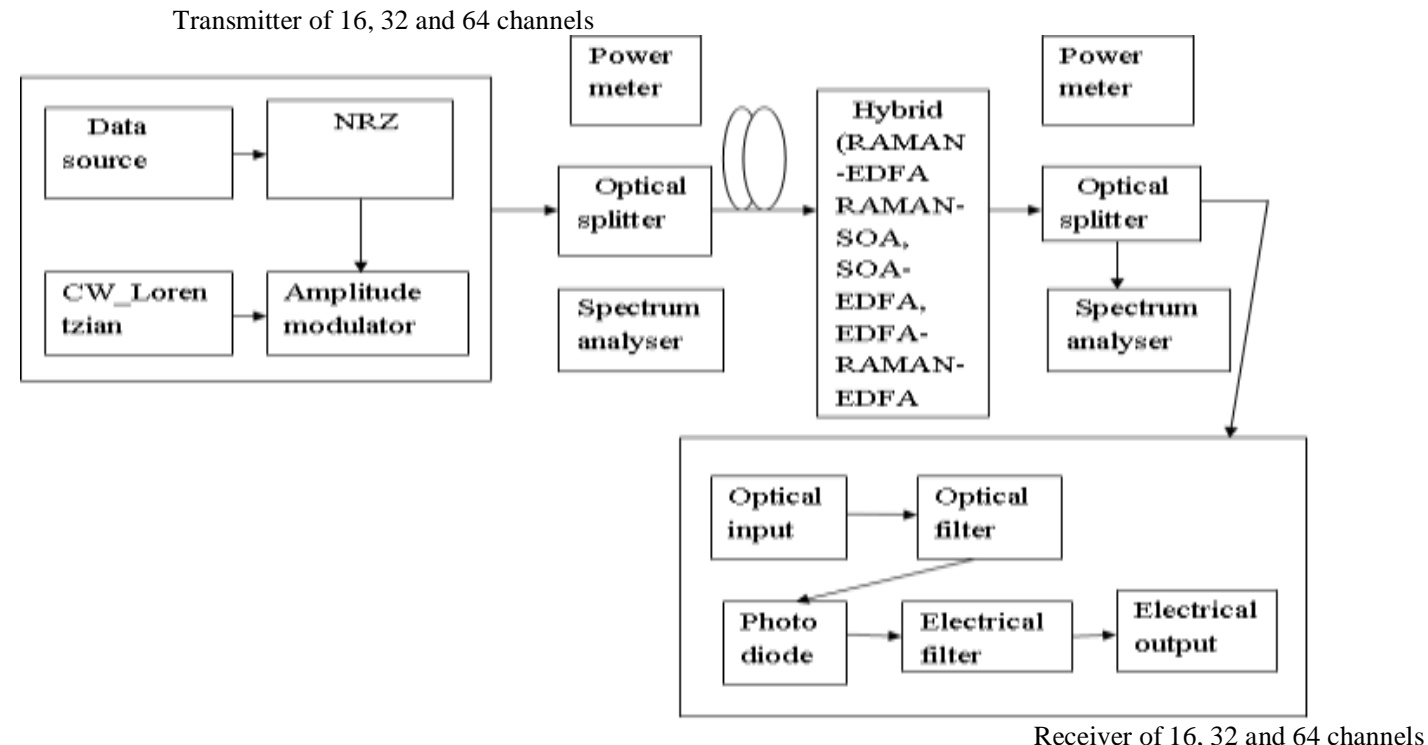

Fig 1: block diagram of simulation setup

\section{RESULTS AND DISCUSSIONS}

The performance of different hybrid amplifiers is compared at different distances. As we increase the distance, the output power decreases simultaneously. Different components have different operational parameters. The comparison of different hybrid amplifiers at different channels is discussed.
In order to observe the performance of different amplifiers (Raman-Edfa, Raman-Soa, Soa-Edfa, Raman-Edfa-Raman), the quality factor versus transmission distance graph is plotted. 


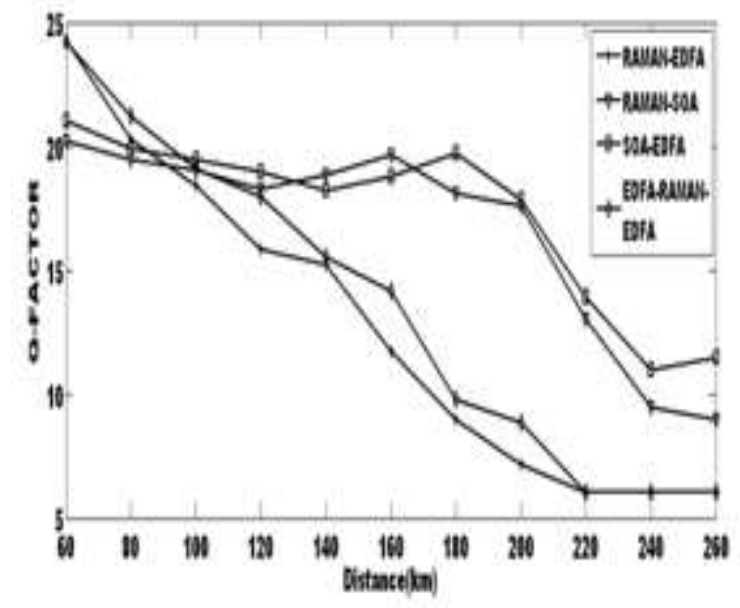

Fig 2 Distances vs. Quality Factor for 16 channels

As we increase the transmission distance from $60 \mathrm{~km}$ to 260 , the quality factor decreases because of the crosstalk and carrier density fluctuation in SOA. The variation in different optical amplifier at distance $60 \mathrm{~km}$ is 24.273 for Raman-Edfa, 20.206 for Raman-Soa, 21.019 for Soa-Edfa, and 24.249 for Raman-Edfa-Raman. The variation in Quality Factor for different optical amplifier at distance $220 \mathrm{~km}$ is 6.020 for Raman-Edfa, 13.020 km for Raman-Soa, 13.904 for Soa-Edfa, 6.020 for Raman-Edfa-Raman.

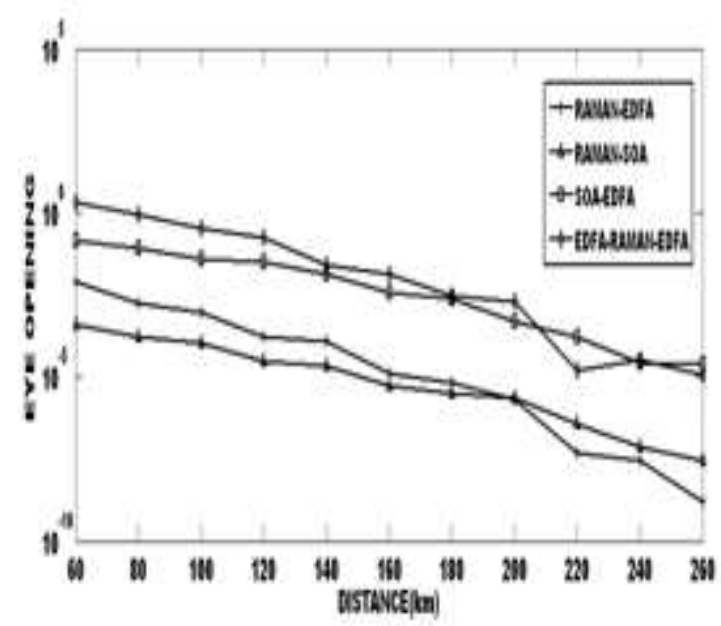

Fig 3 Distances vs. Eye Opening for 16 channels

In order to observe the performance of different hybrid amplifiers, the eye opening versus transmission distance are shown in figure 3.This graph shows that as we increase the transmission distance from 60 to $260 \mathrm{~km}$, the eye opening increases simultaneously from .007676 to $1.61481 \times 10^{-09}$. The eye opening from different amplifiers verses transmission distance is shown in figure 3.Large eye opening means less BER and good communication

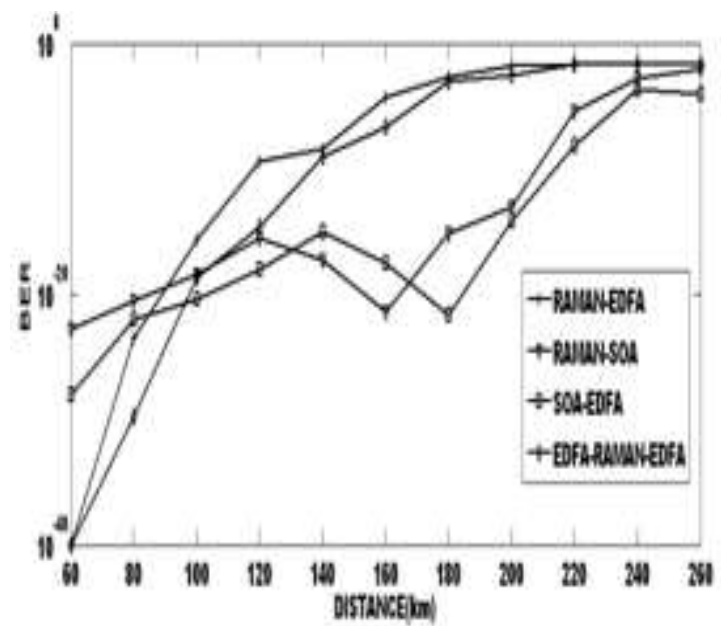

Fig 4 Distances vs. Ber for 16 channels

As shown in figure 3.BER increases with distance from $10^{-}$ 40 to .0227501 for Raman-Edfa and $10^{-23}$ to $10^{-06}$ for RamanSoa. Further for Soa-Edfa, BER increases from $10^{-29}$ to $10^{-05}$. The acceptable bit error rate (BER) for optical transmission is $1 \times 10^{-10}$. Means there is more distortion in the detected signal. The BER versus transmission distance is shown in figure. It is observed that by increasing the transmission distance from 60 to $260 \mathrm{~km}, \mathrm{BER}$ is also increasing.

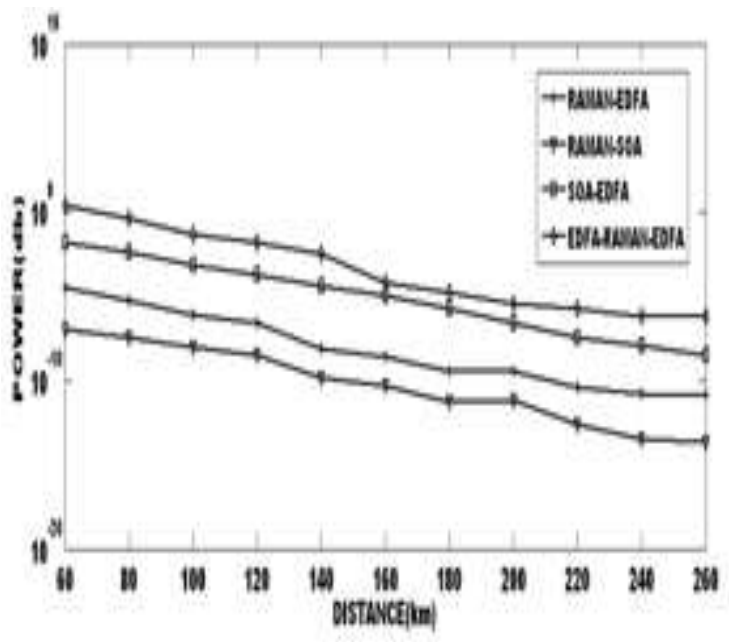

Fig 5 Distances vs. Power for 16 channels

As shown in fig 5 the power versus distance, power decreases with distance from $.112 \times 10^{-06}$ to $.231 \times 10^{-13} \mathrm{db}$ for Raman-Edfa,. $314 \times 10^{-04}$ to .128 X $10^{-10}$ for Raman-Soa,. $143 \times$ $10^{-01}$ to $.361 \times 10^{-08}$ for Soa-Edfa and $.240 \times 10^{01}$ to $.712 \times 10^{-}$ ${ }^{06}$ for Raman-Edfa-Raman for 16 channel The acceptable power for optical transmission is $10 \mathrm{db}$. It is observed that by increasing distance from 60 to $260 \mathrm{~km}$, power is also decreasing. 


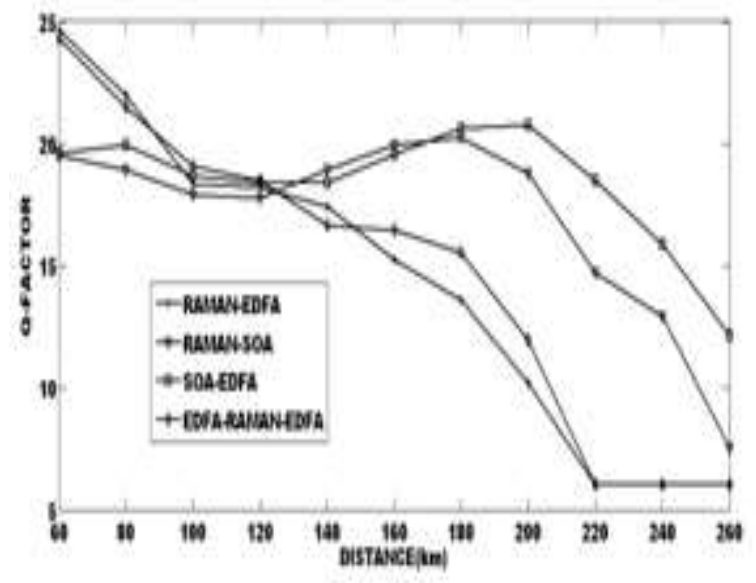

Fig 6 Distance vs. Quality factor for 32 channels

In order to observe the performance of different hybrid amplifiers, the quality factor versus transmission distance are shown in figure 6.This graph shows that as we increase the transmission distance from 60 to $260 \mathrm{~km}$, the quality factor decreases simultaneously. The quality factor decreases from 24.645 to $6.020 \mathrm{~dB}$ for Raman-Edfa,19.495 to7.52 db for Raman-Soa, 19.58 to12.157 for Soa-Edfa and 24.32 to 6.020 for Raman-Edfa-Raman.

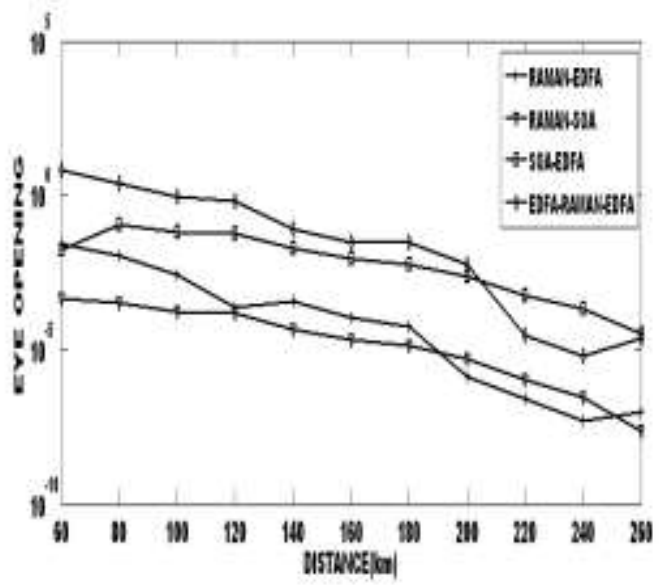

Fig 7 Distances vs. Eye Opening for 32 channels

In order to observe the performance of different hybrid amplifiers, the eye opening versus transmission distance are shown in figure 7. Large eye opening means less BER and good communication. This graph shows that as we increase the transmission distance from 60 to $260 \mathrm{~km}$, the eye opening increases simultaneously

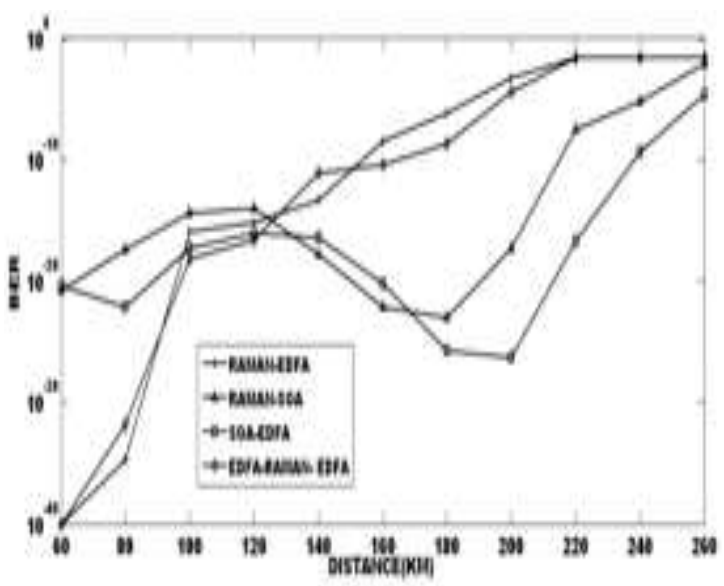

Fig 8 Distances vs. Ber for 32 channels

The BER versus transmission distance for different dispersion is shown in figure. It is observed that by increasing the transmission distance from 60 to $260 \mathrm{~km}$, BER is also increasing. The acceptable bit error rate (BER) for optical transmission is $1 \times 10^{-10}$.

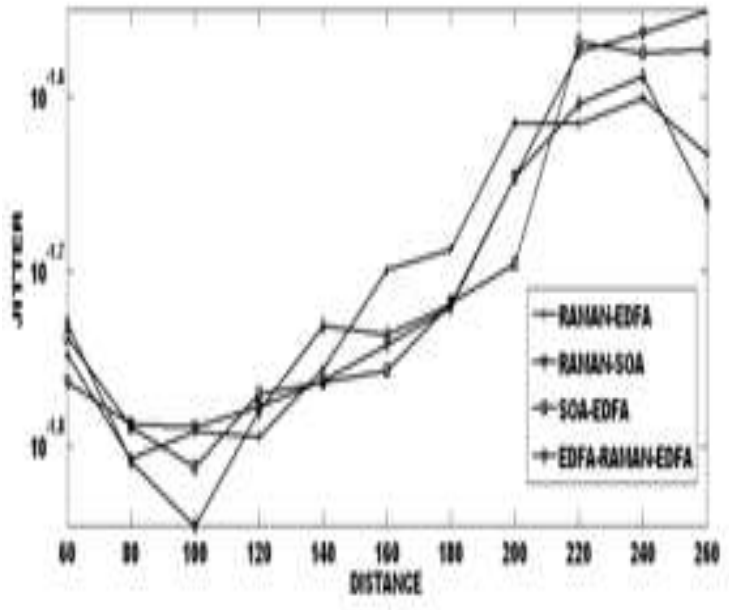

Fig 9 Distances vs. Jitter for 32 channels

In order to observe the performance of different hybrid amplifiers, the jitter versus transmission distance are shown in figure 3.This graph shows that as we increase the transmission distance from 60 to $260 \mathrm{~km}$, the jitter decreases and then increases simultaneously. 


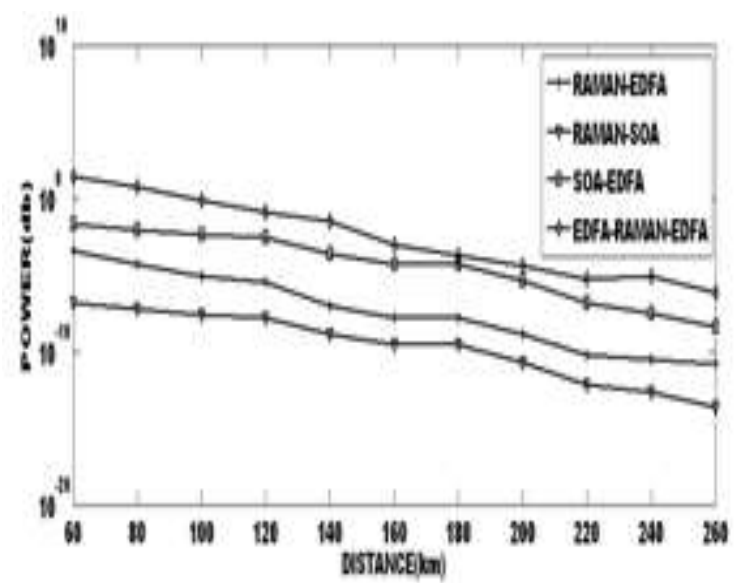

Fig 10 Distances vs. Power for 32 channels

The power versus distance is shown in figure 4.Power decreases with distance from $.350 \times 10^{-03} \mathrm{db}$ to $.163 \times 10^{-10}$ for 32 channels for Raman-Edfa. It is because of non linearities and losses in the channel. The acceptable power for optical transmission is $10 \mathrm{db}$. It is observed that by increasing distance from 60 to $260 \mathrm{~km}$, power is also decreasing.

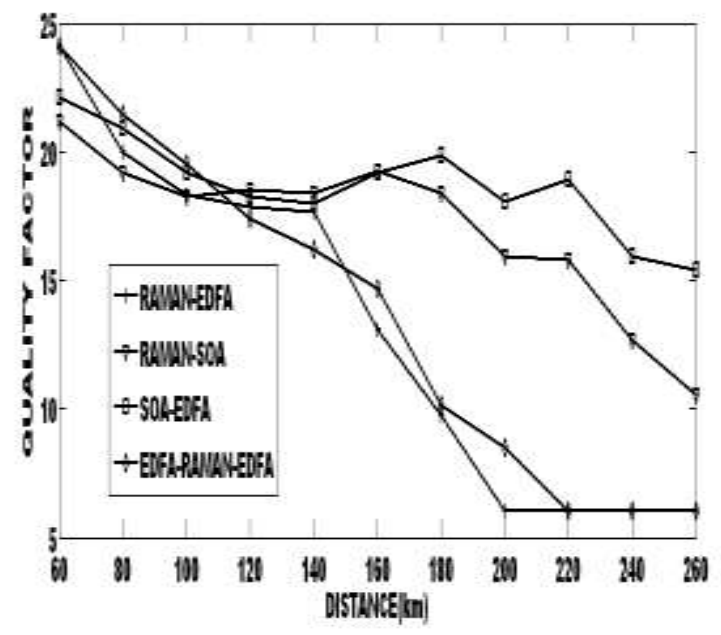

Fig 10 Distances vs. Quality Factor for 64 channels

This graph shows that as we increase the transmission distance from 60 to $260 \mathrm{~km}$, the quality factor decreases simultaneously because of the carrier density fluctuation and crosstalk in SOA. The quality factor decreases from 24.117 to $6.020 \mathrm{db}$ for Raman-Edfa, 21.184 to10.521 db for Raman-Soa, 22.105 to 15.321 for Soa-Edfa and 24.125 to 6.020 for RamanEdfa-Raman.

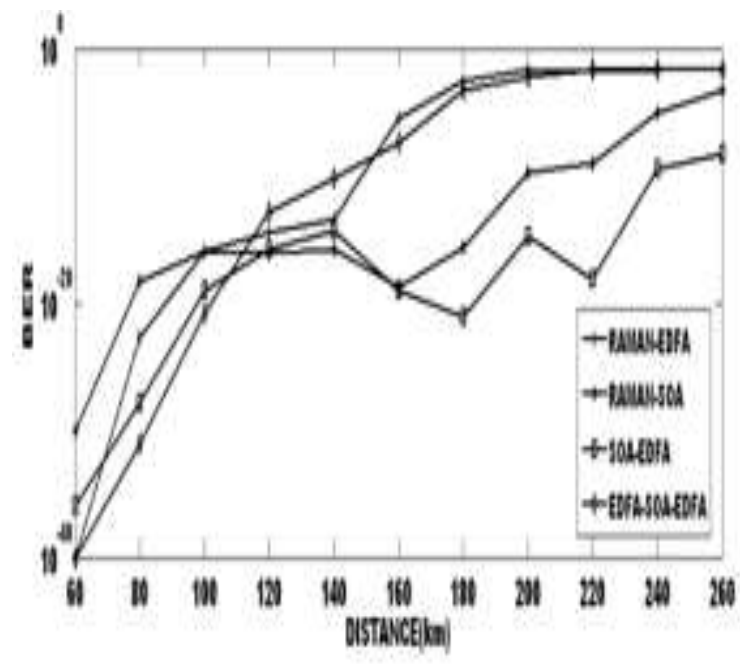

Fig 11 Distances vs. Ber for 64 channels

The BER versus transmission distance for different dispersion is shown in figure. It is observed that by increasing the transmission distance from 60 to $260 \mathrm{~km}$, BER is also increasing. As shown in figure 11, BER increases with distance from $10^{-40}$ to .0227501 for Raman-Edfa and $10^{-31}$ to .000488 for Raman-Soa. Further for Soa-Edfa, BER increases from $10^{-37}$ to $10^{-09}$

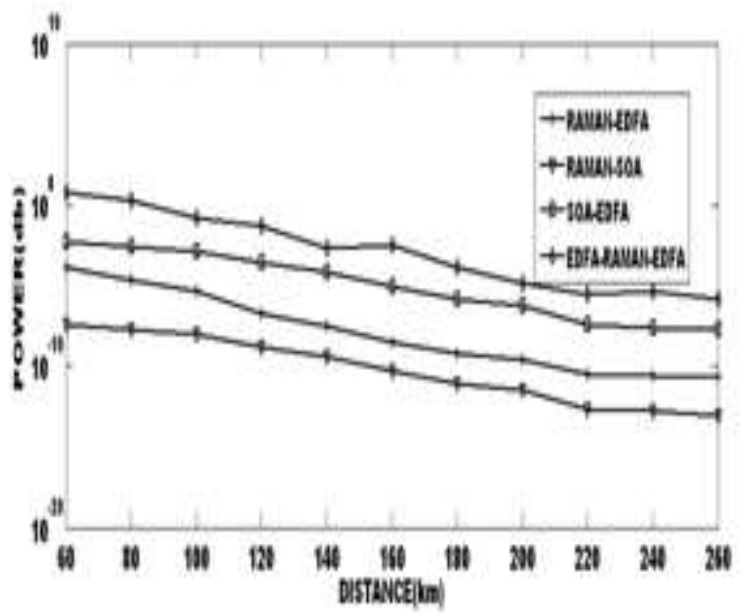

Fig 12 Distances vs. Power for 64 channels

The power versus distance is shown in figure 12.Power increases with distance from $.57 \times 10^{01}$ to $.134 \times 10^{-05} \mathrm{db}$ for 64 channels. The acceptable power for optical transmission is 10 $\mathrm{db}$. It is observed that by increasing distance from 60 to 260 $\mathrm{km}$, power is also decreasing. 


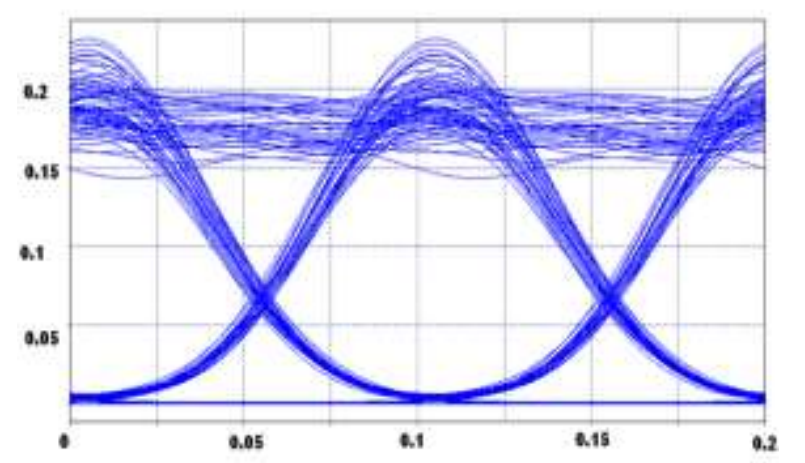

Figure 13(a) SOA-EDFA, 16 channels for Distance $=60$

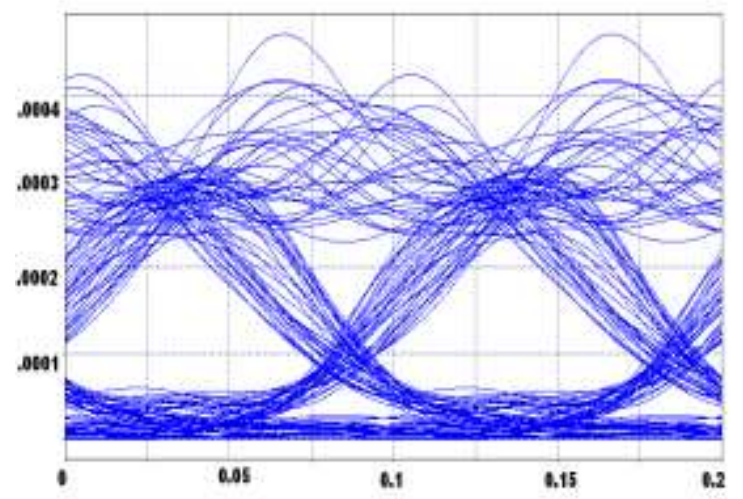

Figure 13(b) SOA-EDFA, 16 channels for Distance $=220 \mathrm{kms}$

It is observed from the simulation result that maximum eye opening is obtained from SOA-EDFA is $1.37 \times 10^{-1}, 4.6 \times 10^{-4}$ for $\mathrm{D}=60 \mathrm{~km} \quad$ and $\mathrm{D}=220 \mathrm{kms}$ respectively.

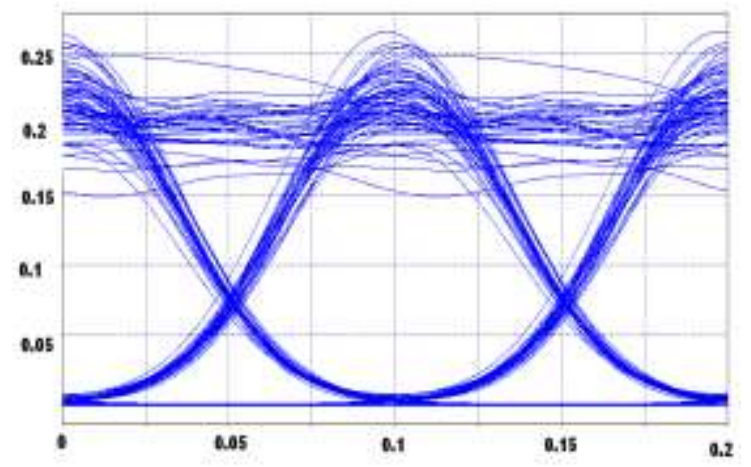

Figure 14(a) SOA-EDFA, 32 channels for Distance $=60 \mathrm{kms}$

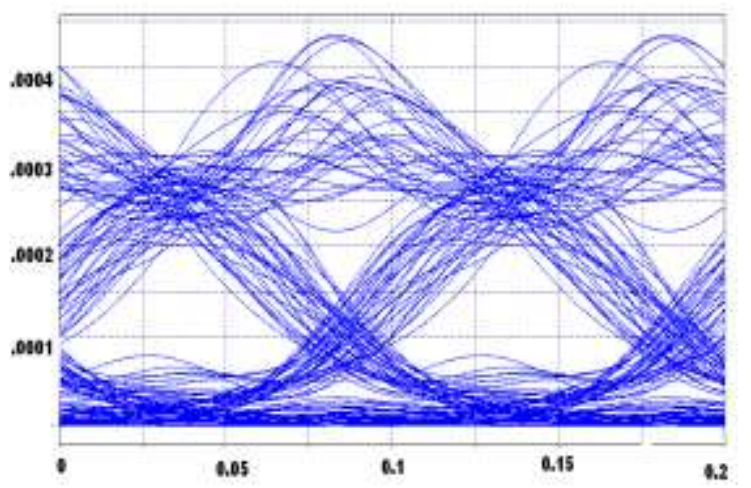

Figure 14(b) SOA-EDFA, 32 channels for Distance $=240 \mathrm{kms}$
Eye diagram of signal for 32 channel of SOA- EDFA at 60 $\mathrm{kms}$ and $240 \mathrm{kms}$ distance shown in figure. The eye opening for distance $60 \mathrm{~km}$ is $1.7 \times 10^{-1}$ and for $240 \mathrm{~km}$ is $2.1 \times 10^{-4}$ respectively.

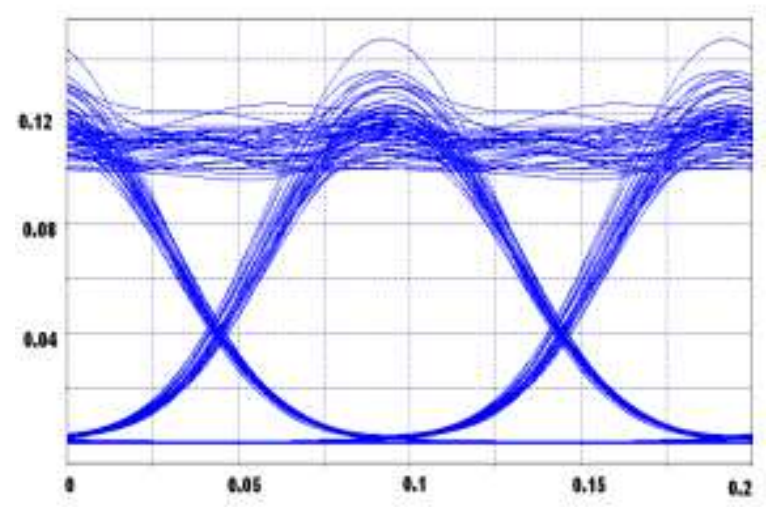

Figure 15(a) SOA-EDFA, 64 channels for Distance $=60 \mathrm{kms}$

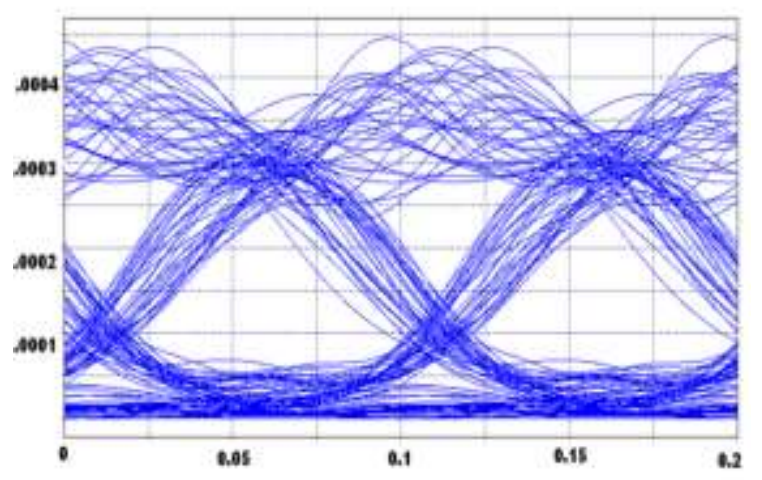

Figure 15(b) SOA-EDFA, 64 channels for Distance $=260 \mathrm{kms}$

Eye diagram of signal for SOA-EDFA at $60 \mathrm{~km}$ and 260 distances for 64 channels is shown in figure 3.13. The eye opening for distance $60 \mathrm{~km}$ is $9.3 \times 10^{-2}$ and $1.7 \times 10^{-4}$ respectively.

\section{CONCLUSION}

The performance of different hybrid amplifiers is compared at different distances. The result shows that SOA combined with EDFA allows the increase of maximum reachable distance and span length. In this SOA-EDFA showed good performance at all the channels as it can travel max distance. The proposed configuration consists of 16, 32 and 64 channels. Hybrid SOAEDFA are enabling and promising for future as it can travel the maximum distance. It is observed through simulation that RAMAN-EDFA provides the best performance in terms of quality factor, ber, eye opening and jitter. But as the distance increases, the quality factor and power degrades whereas ber increases. In case of SOA-EDFA, covers maximum distance i.e. 220,240 and $260 \mathrm{kms}$ with variations in channel.

\section{REFERENCES}

[1] Manjit Singh, Ajay K. Sharma, R.S.Kaler 'Investigations on order and width of RZ super Gaussian pulse in pre-, post- and symmetricaldispersion compensated $10 \mathrm{~Gb} / \mathrm{s}$ optical communication system using standard and dispersion compensating fibers', Optik -International Journal for Light and Electron Optics, Vol 121, Issue 7, Apr 2010, Pg 609-616. 
[2] Manjit Singh, Ajay K. Sharma, R.S.Kaler 'Optimizing 10 Gbps optical communication system with duty cycle selection of return to zero pulse', Optik - International Journal for Light and Electron Optics, Vol 119, Issue 8, 16 Jun 2008, Pg 359-364.

[3] D.P, Mitchel J.E, 'A 10-Gb/s 1024-Way-Split 100-km Long-Reach Optical-Access Network', journal of lightwave technology, Vol 25, Issue 3, 2007, Pg 685-695.

[4] A. Jourdan, F. Masetti, M. Garnot, G. Soulage, M. Sontom, Design and implementation of a fully reconfigurable all-optical cross connect for high capacity multi wavelength transport networks, J. Lightwave. Tech. 14 (6) (1996) 1198-1206

[5] Biswanath Mukherjee, 'Optical WDM Networks', University of California, pg 15-18

[6] G.P.Aggarwal, ' Fibre Optic Comm.', John Wiley and Sons, New York, pg 226

[7] A.Carena,V.Curri and P.Poggiolini, 'On the Optimization of Hybrid
Raman/Erbium-Doped Fiber Amplifiers', Vol. 13, NO. 11, Nov 2001, pg 1170-1172

[8] Chien-Hung Yeh, Kuo Hsiang Lai, Ying Jie HUANG Chien-Chung LEE and Sien CHI. ' Hybrid L-Band Optical Fiber Amplifier Module with Erbium-Doped Fiber Amplifiers and Semiconductor Optical Amplifier', Vol. 43, No. 8A, 2004, pg 5357-5358

[9] Ju Han Lee, You Min Chang, Young Geun Han, Haeyang Chung, Sang Hyuck Kim, and Sang Bae Lee. A Detailed Experimental Study on Single-Pump Raman/EDFA Hybrid Amplifiers: Static, Dynamic and System Performance Comparison, Vol 23, I11, 2005, pg 3484-3493

[10] Seung Kwan Kim, Sun Hyok Chang, Jin Soo Han, and Moo Jung Chu. Design of Hybrid Optical Amplifiers for High Capacity Optical Transmission, Vol 24, No2, 2002, pg-81-96

[11] Tadashi Sakamoto, Shin-ichi-Aozasa, Makoto Yamada, and Makoto Shimizu. Hybrid Fiber Amplifiers Consisting of Cascaded TDFA and EDFA for WDM Signals, 2006, Vol 24, I6, pg-2287-2295 